\title{
Optical Fiber Coupler Analysis Using Daubechies Wavelets
}

\author{
P. C. L. da Silva ${ }^{1}$ (D), R. R. de Melo ${ }^{2,3}$ (D) J. P. da Silva ${ }^{3}$ (D) \\ ${ }^{1}$ P. C. L. da Silva, Department of Natural Sciences Mathematics and Statistics, Federal Rural University of the \\ Semi-Arid, Mossoró,Brazil, linhares@ufersa.edu.br, \\ ${ }^{2}$ R. R. de Melo, Computer Engineering, Federal University of Ceará, Sobral, Brazil, reuber.regis@sobral.ufc.br \\ ${ }^{3}$ R. R. de Melo and J. P. da Silva, Department of Electrical Engineering, Federal University of Rio Grande do \\ Norte, Natal, Brazil, patroc@ct.ufrn.br
}

\begin{abstract}
This article presents the theoretical analysis of a signal coupling between a proposed Photonic Crystal Fiber (PCF) and a Conventional Fiber with Step Index (CFSI) system. In this analysis, the Finite Element Method (FEM) and Beam Propagation Method (BPM) are applied together with Daubechies wavelets as basis function and described in the mathematical formulation. The simulations were performed casting the PCF signal into CFSI core using the PCF refractive index. For simulation purposes, the cladding material refractive index was set as $1.5 \%$ lower than the core index. The application of Daubechies wavelets as basis functions in BPM analysis presented insertion loss around $3.8 \%$ during the fiber coupling.

Index Terms - Daubechies Wavelets, PCF, CFSI.
\end{abstract}

\section{INTRODUCTION}

An important effect on the transmission of signals through optical fibers is the power transfer related to fiber coupling, which is necessary for the most diverse applications. Among the various classes of optical fiber couplers, the couplers based in Photonic Crystal Fiber (PCF) present great research interest due particular spectral features, high performance and great directionality potential applied in high-rate ultra-broadband communication systems [1]-[3]. PCF has revolutionized optical fiber technology due to new degrees of freedom for fiber design, fabrication, and applications which could be achieved. Regarding manufacturing, it is possible to use different materials and geometries, according to the desired propagation mechanism, in a large range of wavelengths [4].

Multi-resolution analysis with wavelets developed by Stephane Mallat [5] is a technique that allows the visualization of signals at various resolution levels, in this work it represents the spectral content of optical fiber signals. Here, the implementation of the basis functions with wavelets was useful for visualizing possible discontinuities, speed up the convergence of the method performed by the simulations, and mapping the optical signal energy levels at different resolution levels.

The coupling structure proposed in this work requires a powerful numerical tool to allow a deeper understanding of the electromagnetic phenomena associated with the interpretation of signal propagation. Thus, an important computational tool applied in the propagation analysis in photonic structures is the Vector Beam Propagation Method (VBPM) [6], which combined with basis functions 
of the wavelets type allows the visualization of the signal variations along the propagation considering the effects caused by the PCF holes and the signal evolution when coupled to the conventional fiber.

In this article, the VBPM was modified to accept the elementary matrices coefficients got from Daubechies wavelets applied on a free scalar field in one dimension, where the spatial derivatives couple the degrees of freedom on different scales, although the method applies to more complex field theories. In the formulation, Perfectly Matched Layers (PML) [7], [8] were used to bound the computational domain.

In the next section, the finite element formulation is briefly explained, Daubechies' theory of wavelets instruction is described in section III, the coupler design and the numerical results are shown in section IV, and finally, the conclusions of this article are presented.

\section{Finite ELEMENT Method}

\section{A. Helmholtz Equations}

The finite element approach for the analysis of electromagnetic propagation characteristics in optical fibers has been studied and applied successfully in several types of structures. Generally, software-based in Maxwell's electromagnetism are used to simulate communication systems involving optical fibers. Therefore, the fiber structural parameters turn to be essential to ensure the results precision. Usually, the computational performance depends on the approximations applied during the mathematical formulation development. In this article, to proceed with the formulation, the procedure was started from Helmholtz equation for the magnetic field [4]:

$$
\nabla \times(\overline{\bar{k}} \nabla \times \vec{H})-k_{0}^{2} \vec{H}=0
$$

Where $\overline{\bar{k}}=\frac{1}{\overline{\bar{\varepsilon}}}$ and $\overline{\bar{\varepsilon}}$ represent the relative permissiveness tensor, $\nabla \times$ is the curl operator, $k_{0}$ is the free space wavenumber, $\omega$ is the angular frequency and $c$ is the speed of light in free space. After some mathematical manipulations and assuming that the electromagnetic fields ranging slowly along the z-propagation direction, (1) may be rewritten as follows:

$$
[A]\{\varphi\}=n_{\text {eff }}^{2}[B]\{\varphi\}
$$

Where $[A]$ and $[B]$ are sparse and complex matrices. Equation (2) is efficiently solved through the interaction of subspaces method. Equation (2) was obtained from (1) and the details are presented in [6]. In this work, for (2), $n_{e f f}$ is the effective refractive index of the structure when it converges to a modal solution.

\section{B. Elementary Matrices}

After the mathematical development and application of the Finite Element Method (FEM) for the discretization of the domain in which (1) is defined, followed by the application of the Galerkin Method, the author in [9] uses Daubechies wavelets in the calculation of the elementary matrices to the Beam Propagation Method (BPM). Then, the basis functions were approximated using Daubechies wavelets for representing the behavior of the magnetic field inside the elements. 
In the next section, we present a brief description of the elementary matrices, using generation moment function for Daubechies wavelets.

\section{DAUBEChIES WAVELETS APPLIED TO THE VBPM}

Daubechies wavelets are functions that can be applied to generate analysis, decompose, or even obtain information from a dataset. This analysis is usually performed by orthonormal wavelet basis as the Fourier basis. Daubechies wavelets do not have an analytical expression that defines their terms. However, these terms are evaluated using the recursive relations below,

$$
\begin{gathered}
\varphi(x)=\sum_{m=0}^{N-1} a_{m} \varphi(2 x-m) \\
\psi(x)=\sum_{m=2-N}^{1}(-1)^{m} a_{1-m} \varphi(2 x-m),
\end{gathered}
$$

where $N$ is the wavelet kind, $m$ is the translation, $a_{m}$ and $a_{1-m}$ are the filter coefficients. The scale function $\varphi$ has support in the range $[0, N-1]$ with energy concentrated in this range. In general, a function $f(x)=x^{k}$ can be represented by the relation (5) used a basis $\varphi$ of wavelets.

$$
x^{k}=\sum_{m=-\infty}^{+\infty} c_{m} \varphi\left(2^{j} x-m\right)
$$

The terms $c_{m}=\frac{M_{m}^{k}}{2^{j k}}$ are called moments of the scale function $\varphi$ concerning the function $x^{k} . M_{m}^{k}$ is the moment of the father wavelet $\varphi, m$ is the translation, $k$ is the degree of the function $x^{k}$ to be approached, and $j$ the wavelet resolution.

In order to apply Daubechies wavelets in this work, the calculation of Moment Generating Function (MGF) performed in [9] is needed. The MGF is crucial to calculating the approximation of a polynomial function using Daubechies wavelets. MGF's expression is shown below,

$$
M_{m}^{k}=\left\{\begin{array}{c}
\frac{1}{2\left(2^{k}-1\right)} \sum_{r=0}^{k-1}\left(\begin{array}{l}
k \\
r
\end{array}\right) \sum_{s=0}^{N-1} a_{s} s^{k-r} M_{0}^{r}, \text { if } m=0 ; k \neq 0 \\
\sum_{r=0}^{k}\left(\begin{array}{l}
k \\
r
\end{array}\right) m^{k-r} M_{0}^{r}, \text { if } m \neq 0 ; k \neq 0 \\
1, \quad \text { if } \quad k=m=0 .
\end{array}\right.
$$

In which, $a_{s}$ is the Daubechies coefficient, $k$ is the degree of monomial $x^{k}, m$ is the translation of wavelet and $N$ is the wavelet kind.

According to the results shown in [9], the integral $I=\int_{o}^{s} x^{k} d x$ involving Daubechies wavelets can be expressed by,

$$
I=\sum_{m=-\infty}^{+\infty} \int_{0}^{s} \frac{M_{m}^{k}}{2^{j k}} \phi\left(2^{j} x-m\right) d x .
$$

The calculation of the elementary matrices to the (BPM) uses the integral (7) and the transformation 
$\tilde{\psi}$ given by,

$$
\begin{gathered}
\tilde{\psi}(x, y)=(1-0.634 \varphi(x)+0.366 \varphi(x+1)+1.366 \varphi(x+2)-y, \\
0.634 \varphi(x)-0.366 \varphi(x+1)-1.366 \varphi(x+2), \quad y)
\end{gathered}
$$

With, $0 \leq x \leq 1$ and $0 \leq y \leq 1-0.634 \varphi(x)+0.366 \varphi(x+1)+1.366 \varphi(x+2)$.

The expressions in (7) and (8) are fundamental for the analysis of the development of the functions proposed in this work.

\section{COUPLER DESign AND Simulations RESUltS}

\section{A. Coupler Design}

Fig. 1 shows the Conventional Fiber with Step Index (CFSI) overlapped by the PCF, forming the proposed coupler design. The PCF is composed of pure silica with refractive index of 1.46 along with air holes distributed in hexagonal rings form, with defects caused by the removal of air holes to form one extended core. CFSI is a conventional fiber composed of a pure silica core with a radius about $4.0 \mu \mathrm{m}$.

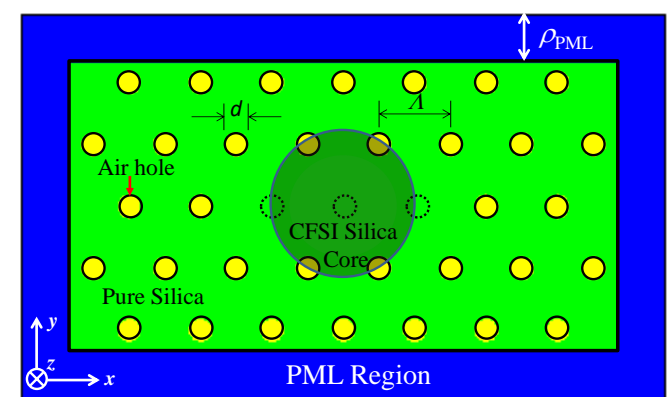

Fig. 1. Proposed Structure.

Recent studies verified that periodic structures could give rise to important phenomena and properties only observed in these types of structures, such as [10] and [11]. For example, the influence that introducing six hexagonal air hole rings into a low refractive index dielectric matrix can cause in photonic gaps, that is frequency bands in which the propagation of light is completely prohibited [11].

The application of Daubechies wavelet in the analysis of these structural models presents high precision because it is a problem that can be originally described through independent variables in space. Due to its ability to decompose frequency and time domain functions, the Daubechies wavelet function applied in this work could identify a high degree of singularities during signal propagation in the PCF.

\section{B. Simulation Results}

The numerical results were obtained from the structure shown in Fig. 1. For the PCF, air holes diameter was set as $d=1.2 \mu \mathrm{m}$ and $\frac{d}{\Lambda}=0.4$, where $\Lambda$ represents the distance between air hole centers. The CFSI has a core with refractive index $n_{C F S I}=1.46$ immersed in a cladding of refractive index equal to 1.44. The CFSI was excited with a signal from PCF with wavelength $\lambda=1.55 \mu \mathrm{m}$, polarized in the y-direction. The computational window was $26 \mu \mathrm{m}$ (x-direction) by $16 \mu \mathrm{m}(\mathrm{y}-$ Brazilian Microwave and Optoelectronics Society-SBMO received 9 Jan 2020; for review 16 Jan 2020; accepted 11 June 2020 Brazilian Society of Electromagnetism-SBMag 
direction) divided into, approximately, 22,000 triangular elements. The propagation of light in PCF occurs due to the total internal reflection phenomenon, which describes light guidance in the solid central core [9]. Therefore, the losses involved in the coupling occur only by insertion, due to different cross-sectional structures. Fig. 2 shows the convergence for effective permittivity according to the signal launched in the CFSI. It is possible to observe a reduction in permissiveness in relation to PCF permissiveness, which can be attributed to insertion loss.

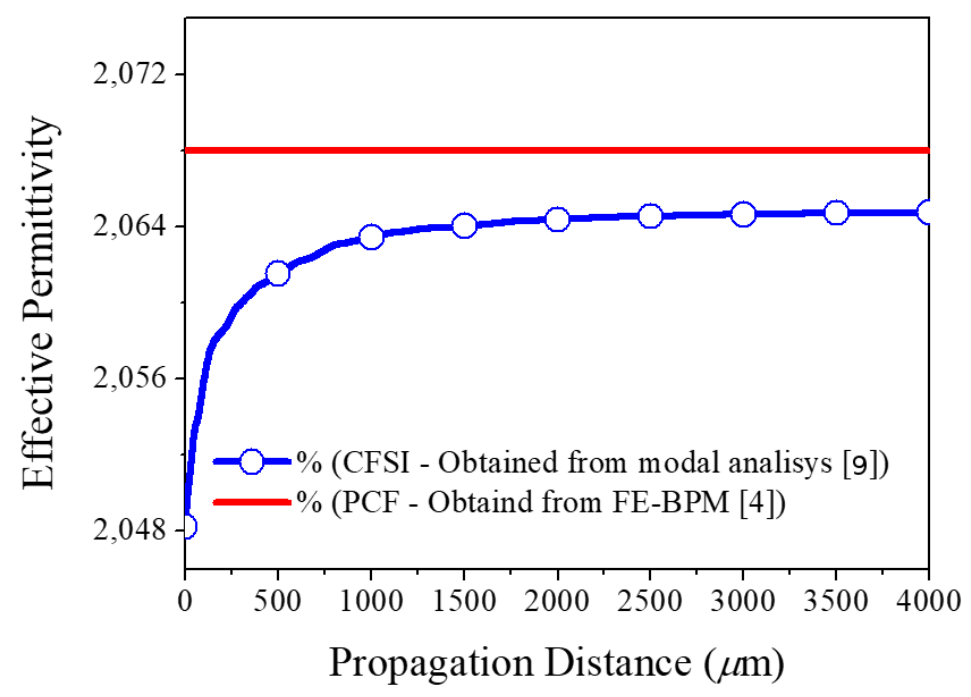

Fig. 2. Variation of the reference refractive index during signal propagation.

The hexagonal PCF has been designed to operate in a single-mode with flattened dispersion wideband at the wavelength range from $1500 \mathrm{~nm}$ to $1580 \mathrm{~nm}$. For the numerical analysis, this wavelength was centered at $1550 \mathrm{~nm}$ where it can be perfectly applied to an optical transmission system.

Fig. 3 and Fig. 4 show the magnetic field distribution, from modal analysis simulation, generated in the polarized PCF in the y-direction. This signal was launched in the CFSI. Fig 3 was obtained using the elementary matrix coefficients by linear approximation, while Fig 4 was obtained using the elementary matrix coefficients obtained by Daubechies wavelet.

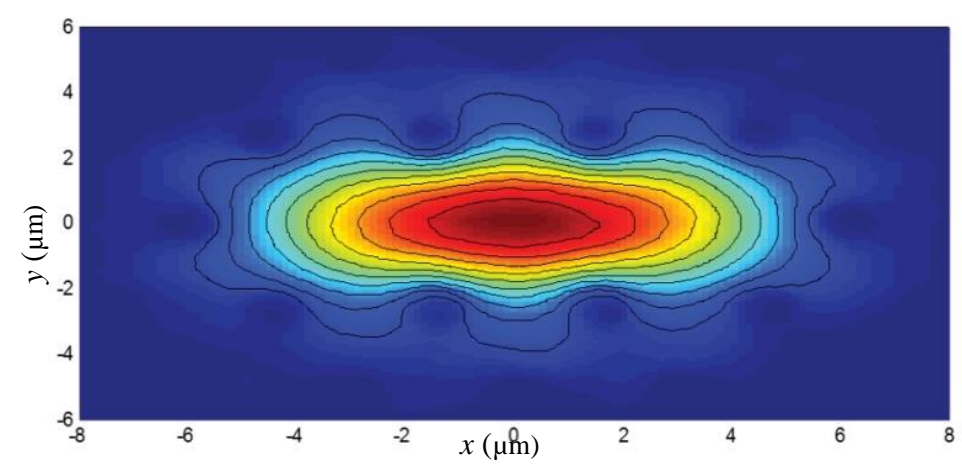

Fig. 3. The y component of CFSI input magnetic field signal performed by elementary matrix with linear approximation. 


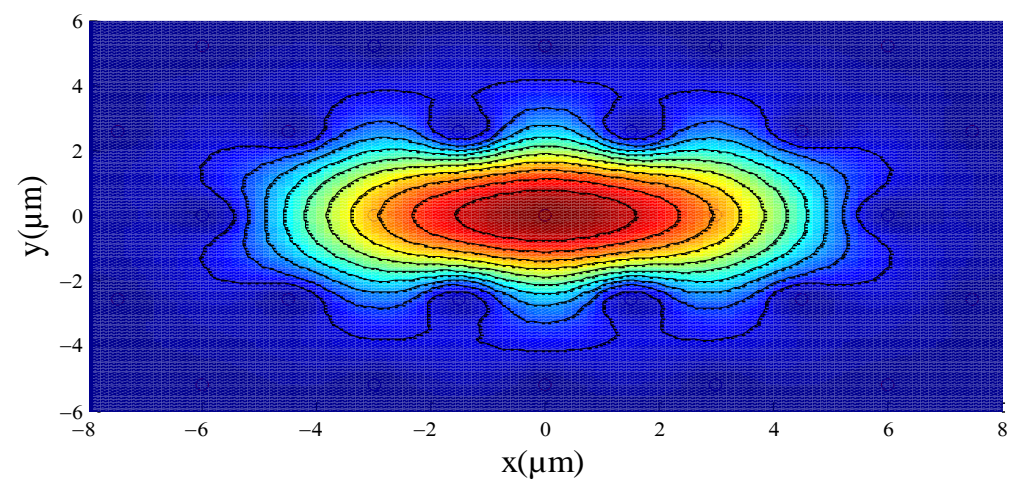

Fig. 4. The y component of CFSI input magnetic field signal performed by elementary matrix Daubechies wavelets coefficients.

Fig. 4 illustrates the potential of Daubechies wavelet in this application, which requires dimensionality reduction to allow an immediate distinction of patterns. The coefficients obtained for the elementary matrix assisted by Daubechies wavelet allowed a good precision of the pattern recognition procedure for the magnetic field. In this simulation, the contribution of the wavelet is to allow the extraction of relevant information from the initially imperceptible propagated signal.

It is possible to observe a greater detail of the magnetic field contour in Fig 4 when compared with the magnetic field contour shown in Fig 3. This detail was possible due the use of Daubechies wavelet basis of kind $N=4,6$, and 8 , which shows a better contour according to the structure of the enlarged core, especially in the center of the figure. Furthermore, it was also possible to obtain a faster energy transfer from PCF to CFSI.

Fig. 5 shows the magnetic field distribution of the y-component, launched in the CFSI, after $500 \mu \mathrm{m}$ propagated, from BPM simulation. This result was obtained with the application of the VBPM described in [6] and the basis of Daubechies wavelets used in [9]. It was possible to obtain a major degree of resolution, guaranteeing the success of the results obtained in this simulation.

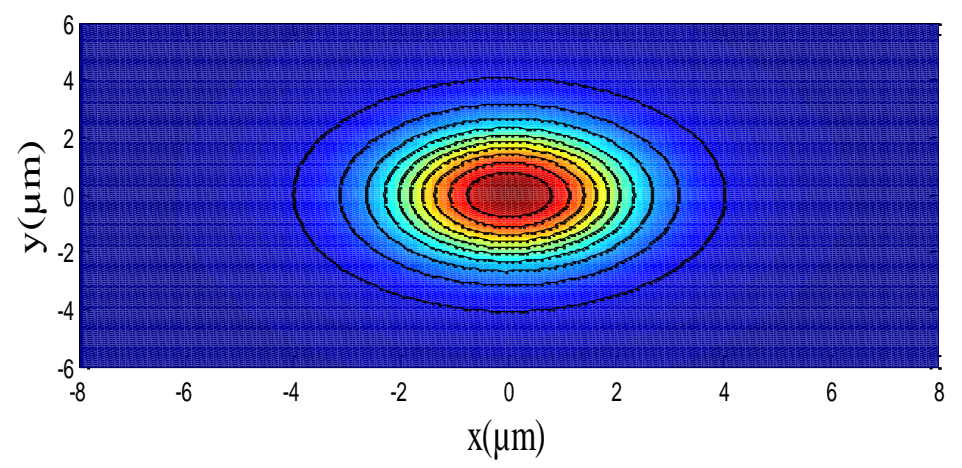

Fig. 5. The y component of the magnetic field after $\mathbf{5 0 0} \mu \boldsymbol{m}$ propagated.

After the convergence, the signal acquires the CFSI format operating at $1550 \mathrm{~nm}$ for a single-mode application. It can be verified the insertion loss that occurs in the resultant energy of the CFSI signal insertion from the PCF. The result obtained in this work, for this parameter, was calculated 
considering the difference between the power launched in the PCF to the CFSI after coupling, that is, after the total transference of the light signal in the PCF to the CFSI.

As the signal propagation occurs along the z-direction by successive reflections, an insertion loss around 3.8\% was observed during the coupling. This type of loss can be attributed to the absorption or derailments between optical fibers contact surfaces. Here, the power in both fibers was calculated using the Pointing Vector theorem.

\section{CONCLUSIONS}

In this work it was presented the application of the Daubechies wavelet to obtain the coefficients of elementary matrices used in the BPM. An investigation of coupling signal between a PCF and a conventional optical fiber was performed. The main advantage of the wavelet application can be attributed to its multi-resolution analysis capability. The Daubechies wavelets applied as basis functions allow visualization of the spectral distribution of the magnetic field with various levels of resolution, improving the result precision. The signal was launched in the PCF and coupled to the CFSI with structural parameters, adjusted to improve its performance as an optical coupler. Low insertion loss was observed during the coupling, as explained in section IV. The insertion loss occurs due to the difference in the cross-section area between the fibers. The results obtained show that the signal launched in the CFSI from the PCF is completely transferred after $500 \mu \mathrm{m}$ of propagation.

\section{ACKNOWLEDGMENT}

The authors are grateful to the CCEN/UFERSA, PPGEEC/UFRN, DEC/UFC by structural support.

\section{REFERENCES}

[1] T. Zhao et al., "Ultrabroadband Polarization-Insensitive Coupler Based on Dual-Core Photonic Crystal Fiber," IEEE Photonics Journal, vol. 9, no. 1, pp 1-10, 2017.

[2] M. Hossain, B. Hossain and Z. Amin, "Small coupling length with a low confinement loss dual-core liquid infiltrated photonic crystal fiber coupler," OSA Continuum, vol. 1, no. 3, pp. 953-962, 2018.

[3] J. P. da Silva, E. G. do Santos and B. S. Marcos T., "Directional Coupler Based on a Photonic Quasi-Crystal Fiber with Extended Cores," Journal of Microwaves, Optoelectronics and Electromagnetic Applications, vol. 17, no. 2 pp. 201207, 2018.

[4] L. Zhengyong et al., "Multifunctional smart Optical Fibers: Materials, Fabrication, and Sensing Application," Photonics, vol. 6. no. 2, pp. 48, Multidisciplinary Digital Publishing Institute, 2019.

[5] S. G. Mallat, "A theory for multiresolution signal decomposition: the wavelet representation," IEEE Transactions on Pattern Recognition and Machine Intelligence, vol. 11, no. 7, pp. 674-693.

[6] J. P. da Silva et al., "Improved Vectorial Finite Element BPM Analysis for Transverse Anisotropic Media," Journal of Lightwave Technology, vol. 21, no. 2, pp. 567-576, 2003.

[7] G. Karagounis, D. De Zutter, and D. V. Ginste, "Full-wave simulation of optical waveguides via truncation in the method of moments using PML absorbing boundary conditions," Optics Express, vol. 24, no. 25, pp. 28326-28336, 2016.

[8] J.P. Berenger, "A Perfectly Matched Layer for the Absorption of Electromagnetic Waves," Journal of computational physics, vol. 114, no. 2, pp. 185-200, 1994.

[9] P. C. L. da Silva et al., "Daubechies Wavelets as Basis Functions for the Vectorial Beam Propagation Method," Journal of Electromagnetic Waves and Applications, vol. 33, no. 8, pp. 1027-1041, 2019.

[10] J. P. da Silva, "Ge-Doped Microstructured Fiber Design by Genetic Algorithm for Directional Coupling," Microwave and Optical Technology Letters, vol. 55, no. 2, pp. 281-285, 2013.

[11] Y. H. LI, W. D. FAN, and Q. Q. SHENG, "A novel photonic quasicrystal fiber with broadband large negative dispersion," Physics Letters, vol. 27, no. 11, pp. 114211, 2010. 